\title{
Electrospun Cellulose Acetate Nanofibrous Mat as the Tissue Scaffold
}

\author{
Miao Cheng ${ }^{1,}$, , Yunfeng Qin ${ }^{1, b}$, Pengfei Qian ${ }^{1, c}$ and Zongyi Qin ${ }^{1, d}$
}

\author{
${ }^{1}$ State Key Laboratory for Modification of Chemical Fibers and Polymer Materials, and College of \\ Material Science and Engineering, Donghua University, Shanghai 201620, China \\ achengmiao91@126.com, ${ }^{\mathrm{b}}$ qinyunfeng2010@sina.com, ${ }^{\mathrm{c}}$ 1136481541@qq.com, \\ phqin@dhu.edu.cn
}

Keywords: Electrospinning; Cellulose acetate; Nanofibrous mat; Tissue scaffold; MG-63 cell Abstract. The cellulose acetate (CA) nanofibrous mat was fabricated by electrospinning under optimized spinning conditions in this work. The morphology, chemical structure, thermal stability and hydrophilic property of as-prepared CA nanofibrous mat were characterized by scanning electron microscopy, Fourier transform infrared spectrometry, thermal gravimetric analysis and water contact angel measurement. Moreover, its cytocompatibility was evaluated through the cultivation of the MG-63 cells. The result shows the CA nanofibrous mat exhibits excellent biocompatibility and possesses a great potential in biomedical application as the tissue scaffold.

\section{Introduction}

Electrospinning as one of the preferred scaffold processing methods for providing scaffolds composed of nano/micro scale fibers has been exhibited great potential applications in tissue engineering, pharmaceutical, defense and security, and environmental engineering and so on [1,2]. Recently, cellulose acetate (CA) as an environmentally degradable material made from the most abundant biopolymer on earth i.e. cellulose has been widely applied in the industrial and biomedical fields due to its high affinity with other substances, tensile strength, regenerative properties, excellent compatibility with human body environment and relatively low cost [1]. Exploring the multi-fold material properties of $\mathrm{CA}$ in the nanoscale regime is an interesting proposition, due to the extremely high surface-to-volume ratio, tunable porosity and good biocompatibility of CA nanofibers. Materials used in this study were CA, which would be electrospun to form nanofibrous mat under optimized spinning condition. The morphology, chemical structure, thermal stability, and hydrophilicity of CA nanofibrous mat would be investigated. Moreover, its comparative cytocompatibility would be evaluated with human MG-63 cells for the potential biomedical application.

\section{Experimental}

Materials. CA with the number average molecular weight of $30,000 \mathrm{~g} / \mathrm{mol}$ and the degree of substitution of acetyl group of 2.4 was purchased from Sigma-Aldrich (USA). Acetone, ethanol and N, N-dimethylacetamide (DMAc) were obtained from Guangzhou Chemical Agent Company, China. Phosphate buffered solution (PBS) was purchased from the Shanghai Guoyao Group Chemical Reagent Co., Ltd. All the materials and reagents were used as received without further purification. The deionized water was used for all the experiments.

Electrospinning. CA was dissolved in 2:1 (w/w) acetone/DMAc mixtures for preparing $15 \mathrm{wt} . \%$ CA spinning solutions. The electrospinning of CA was carried out on a traditional wet electrospinning set-up with the aluminum plate as static metal collector at room temperature. The deliver flow rate of $1 \mathrm{~mL} / \mathrm{h}$ was used at the potential of $20 \mathrm{kV}$, and the distance between needle and collector was $15 \mathrm{~cm}$. The collected electrospun nanofibers were dried under vacuum at room temperature for $24 \mathrm{~h}$ to remove residual solvent.

Characterization. The morphology of the electrospun nanofibrous mat was observed on a Jeol JSM-5600 scanning electron microscope (SEM). Chemical structures of CA raw material and nanofibrous mat were characterized on a Nicolet 8700 Fourier transform infrared (FT-IR) spectrophotometer. The spectra were collected in a spectral range of $4000-400 \mathrm{~cm}^{-1}$ with 64 scans and 
$4 \mathrm{~cm}^{-1}$ resolution. The thermal properties were evaluated using thermogravimetric analysis (TGA, Q500, TA Instruments, USA) in the nitrogen gas atmosphere with the temperature range from room temperature to $600{ }^{\circ} \mathrm{C}$ at a heating rate of $10{ }^{\circ} \mathrm{C} / \mathrm{min}$. For determination of hydrophilicity of CA nanofibrous mat, the contact angle was measured on a Dataphysics OCA40 contact angle analyzer at room temperature. To evaluate the tissue compatibility of CA nanofibrous mat with the potential application for bone tissue regeneration scaffolds, the human MG-63 cells were used [3]. At culture times of 1,2 , and 4 days, the morphologies of adhering MG-63 cells were determined by a fluorescence microscope (Olympus IX71-22FL/PH).

\section{Results and Discussion}

Morphology. It is well known that the volatility of solvents have a significant influence on the formation and morphology of the nanofibers. When a volatile solvent like acetone was selected as the solvent for electrospinning, the tip of the needle was easily blocked by the polymer and pores maybe formed on the nanofiber surface when solvent evaporated. However, when a high boiling point solvent like DMAC was used as the solvent, the fibers would be fused together before drying and even dissolved again because the solvent cannot evaporate completely during electrospinning. That indicated both acetone and DMAc were not a desirable solvent for electrospinning of CA. Therefore a mixture of acetone/DMAc solvent system at the ratio of 2:1 (v/v) was used to dissolve CA and fabricate electrospun nanofibrous mat [4]. Fig. 1(a) gives the SEM image of CA nanofibrous mat. It can be seen that the CA nanofibrous mat exhibits the high porosities with the random array superfine fibers and all nanofibers are smooth with bead-free. The diameter of the fibers is in the range from 590 to $810 \mathrm{~nm}$ and the average diameter is $720 \mathrm{~nm}$ approximately. Fig. 1(b) indicates that the water contact angle for CA nanofibrous was about $123^{\circ}$. The hydrophilic hydroxyl groups on the surface of cellulose were partial substituted by hydrophobic acetyl groups which would result in the decrease of the hydrophilic property of cellulose.
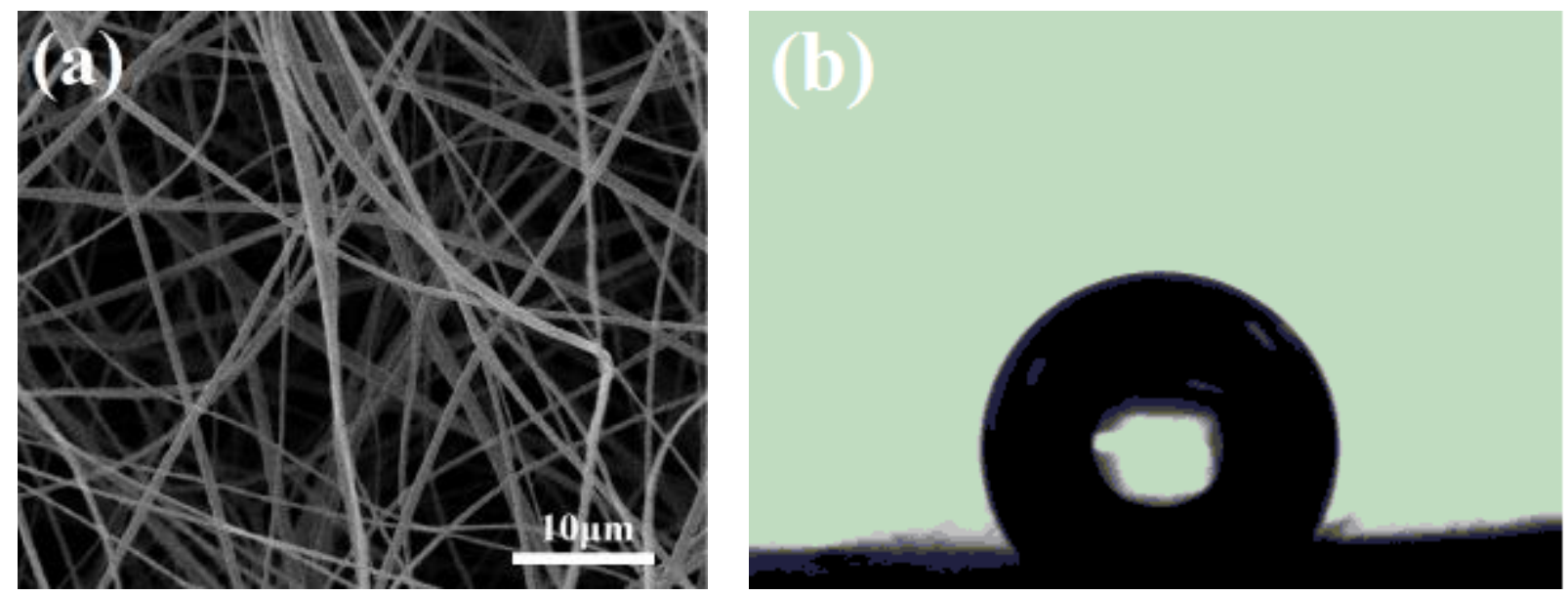

Fig. 1. SEM image (a) and contact angle measurement (b) of CA nanofibrous mat.

Chemical structure. Fig. 2 illustrates FT-IR spectra of the CA raw material and nanofibrous mat. The characteristic absorption peaks at $1049 \mathrm{~cm}^{-1}$ and $1160 \mathrm{~cm}^{-1}$ can be assigned to the asymmetric $\mathrm{C}-\mathrm{O}-\mathrm{C}$ bond stretching from the pyranose ring and $\mathrm{C}-\mathrm{O}-\mathrm{C}$ glycosidic linkage respectively. The characteristic bands such as alkoxyl stretch of the ester at $1235 \mathrm{~cm}^{-1}$, symmetric and antisymmetric bending of methyl groups $\left(-\mathrm{CH}_{3}\right)$ for groups of the acetate substituent at 1375 and $1435 \mathrm{~cm}^{-1}$ could be observed, respectively, and the strong band at $1750 \mathrm{~cm}^{-1} \mathrm{C}=\mathrm{O}$ corresponds to stretching from the acetyl groups [5]. All characteristic bands of CA were observed in CA nanofibrous mat which indicated that electrospinning did not affect the structure of polymer when high voltage was imposed on the polymer. 


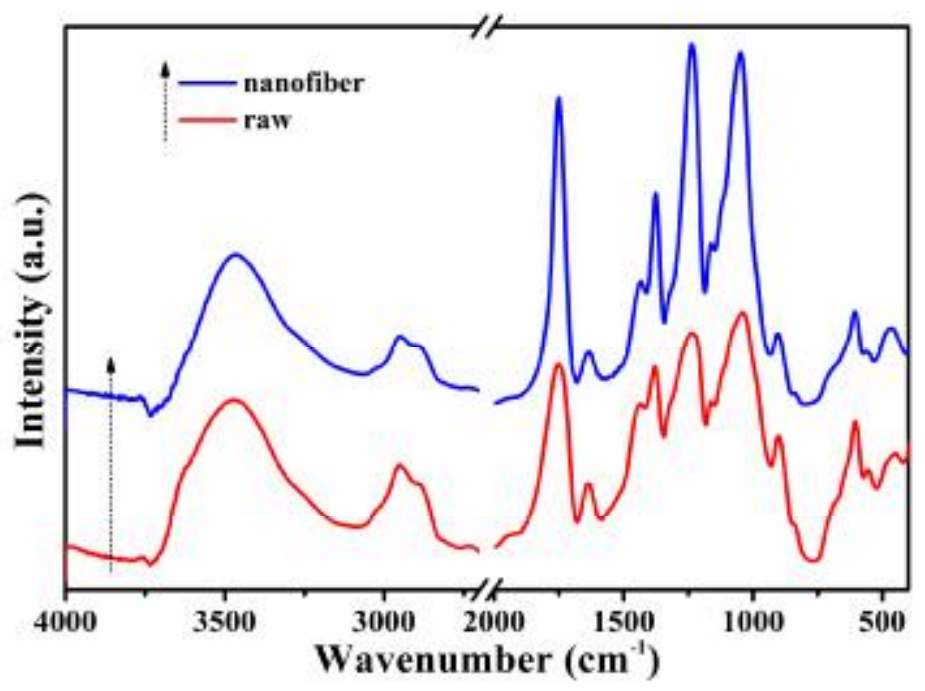

Fig. 2. FT-IR spectra of the CA raw material and nanofibrous mat

Thermal stability. Fig. 3 shows the TGA curves of the CA raw material and nanofibrous mat. It can be found that all the samples showed similar changing trends. The weight loss within the temperature range from 40 to $120^{\circ} \mathrm{C}$ was corresponded to the loss of moisture in the beginning. Then the decomposition took place mainly between $330^{\circ} \mathrm{C}$ and $410{ }^{\circ} \mathrm{C}$, attributing to a cellulose degradation process such as depolymerization, dehydration and the decomposition of glucosyl [6]. All the samples exhibited a maximal rate of mass loss at around $375^{\circ} \mathrm{C}$. The TGA results indicated that only slight influence of the process of electrospining on the thermo-decomposition behavior ofCA as shown in Fig. 3.

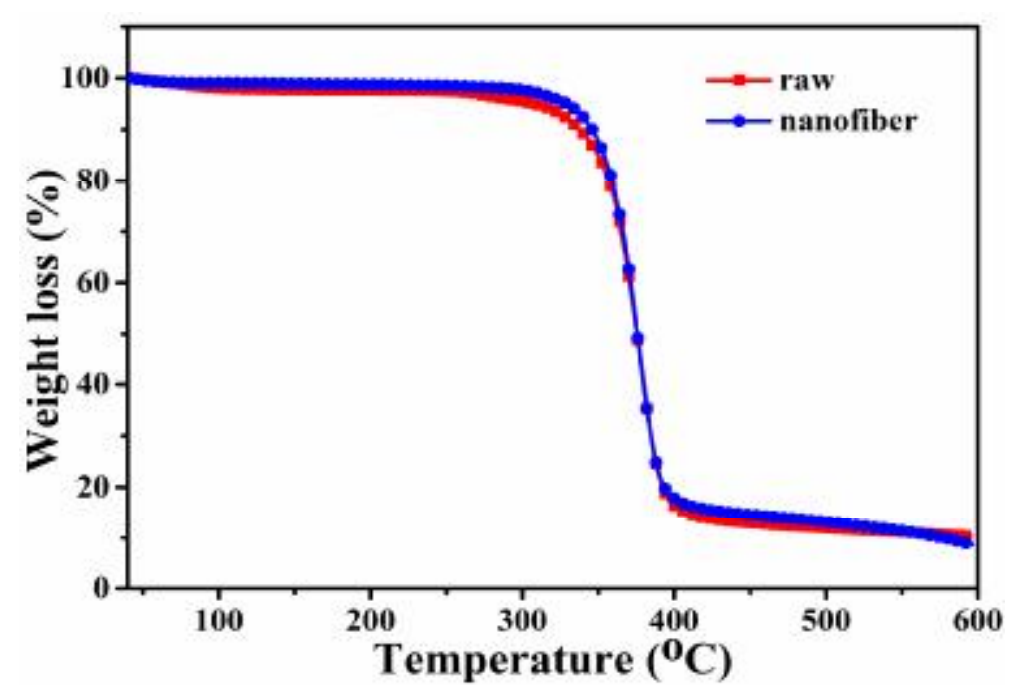

Fig. 3. TGA curves of CA raw material and nanofibrous mat.

Cytocompatibility. Fig. 4 displays the fluorescent images of MG-63 cells cultured on CA nanofibrous mat. The fluorescent images presented in Fig. 4 were used to evaluate the cytocompatibility of $\mathrm{CA}$ nanofibrous mat. The MG-63 cells were represented by the green fluorescence. It is clear that cell density became dense with the increasing of the time. At the first day, cell nuclei emerged and cells begin to climb on the nanofibers but the adhesion was not strong. At the second day, cells adhered and grew well surrounding fibers. After four days' cultivation, the growth of the MG-63 cells had practically completed. The cells had grown mainly along the nanofiber, and covered most of the nanofibrous mats. The result demonstrated that the CA nanofibrous mat possessed excellent biocompatibility and exhibited a great potential application as the tissue scaffold. 


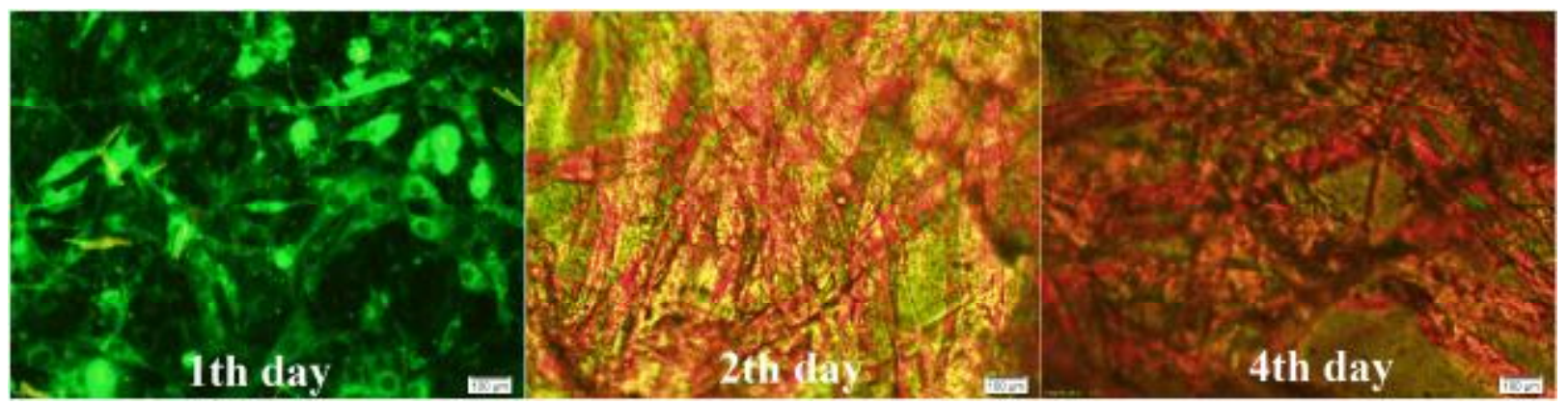

Fig. 4. Fluorescent images of MG-63 cells cultured on CA nanofibrous mat at various times.

\section{Conclusions}

CA nanofibrous mat prepared by electrospinning under optimized spinning conditions. It is found that the as-prepared CA nanofibers exhibit relatively smooth morphology and comparatively uniform diameter (the average diameter of $720 \mathrm{~nm}$ ), and became more hydrophilic (contact angle of about $123^{\circ}$ ) because of the substitution of acetyl groups. Meanwhile, its chemical structure and good thermal stability did not change through the electrospining. More importantly, the excellent comparative cytocompatibility was demonstrated with human MG-63 cells, indicating the great potential of CA nanofibrous mat as the efficient tissue scaffold in biomedical application.

\section{Acknowledgments}

Financial support from the Innovation Research Funds for the Doctoral candidate of Donghua University (15D310606) is gratefully acknowledged.

\section{References}

[1] R. Konwarh, N. Karak and M. Misra: Biotechnology advances Vol. 31 (2013), p. 421.

[2] N. Bhardwaj and S. C. Kundu: Biotechnology advances Vol. 28 (2010), p. 325.

[3] H. Y. Yu, Z. Y. Qin and C. F. Yan: ACS Sustainable Chemistry \& Engineering Vol. 2 (2014), p. 875.

[4] H. Liu and Y. Hsieh: J Polym Sci B: Polym Phys Vol. 40 (2002), p. 2119.

[5] K. Rodríguez, S. Renneckar and P. Gatenholm: ACS applied materials \& interfaces Vol.3 (2011), p. 681.

[6] S. Wu, X. Qin and M. Li: Journal of Industrial Textiles Vol. 44 (2014), p. 85. 